American Journal of Applied Sciences 9 (2): 180-185, 2012

ISSN 1546-9239

(C) 2012 Science Publications

\title{
Performance of Thin-Film as Wavelength Filter in Wdm-Pof Network
}

\author{
Mohd Syuhaimi Ab. Rahman, L.S. Supian, \\ Hadi Guna, Mohd Hazwan Harun and Kasmiran Jumari \\ Department of Electrical, Electronics and Systems Engineering, \\ Faculty of Engineering and Built Environment, \\ University Kebangsaan 43600 UKM Bangi, Selangor, Malaysia
}

\begin{abstract}
Problem statement: Huge capacity for data transmission and bandwidth are becoming a need nowadays. High cost of implementation using optical fibers prevents utilization of the technology as wide as possible. Approach: Wavelength Division Multiplexing (WDM) was a technique that offers the solution for providing better bandwidth. WDM technique allows several signals that have different wavelengths to be transmitted in a single optical fiber to the receiver without any external interference or internal interruption between the signals transmitted simultaneously. WDM technique requires splitting of signals at reaching the receivers and one of the solutions presented in this study will provide a method using inexpensive filtering device that functions efficiently as a demultiplexer that can be develop without any complication. There were a lot of systems that provided demultiplexing techniques in the industry; all the systems are expensive to be implemented. Here the study provides an effective and easy to construct demultiplexer by using colored thin films. The WDM-POF system studies the performances of thin-films to be used as demultiplexer and red thinfilms will be focused. Results: In theory, the thin-films will filter out any undesired signals that pass through the fiber and the desired signal will be carried to the end-receiver. The effectiveness of each red thin- films were characterized by analyzing the insertion losses $(\mathrm{dBm})$ and power output $(\mu \mathrm{W})$. Characterization analysis in this study includes the characterization test of using the same sources towards different types of red thin-films to obtain the best film that could filter out any undesired wavelength and let only the desired wavelength to be transmitted at high percentage. An initial stage of selecting different colored films by analyzing the energy spectrum distribution curves for the potential red thin- films will then be characterized where red, blue and green LED sources were injected through the films. Small insertion loss of the signal transmitted for the same wavelength of the source and high power output will be preferred. High insertion loss and small power output are preferred for filtering out the undesired wavelengths. Comparison and characterization analysis through the samples and graph simulations provides the efficiency of the thin films that were used in the optical WDM-POF short-haul communication system. Conclusion: Optimum performance can be observed through the end-receivers that have been connected to the device developed such as the Ethernet connectivity and the result of video image received the signals as desired.
\end{abstract}

Key words: Color filter, short distance, characterization analysis, undesired signals, optimum performance, ethernet connectivity, image received, optical fibers, communication system

\section{INTRODUCTION}

Fiber-optic system has its own advantages where in communication purposes, the transmission bandwidth or the capacity of information carrying is much bigger than that of conventional transmission media such as coaxial copper cables, wide-band radio or microwave systems. Small size and light weight of optical fibers coupled with low transmission loss, commonly around $0.2 \mathrm{db} \mathrm{km}^{-1}$, in such a way that decrease the cost as well as the requirement for plethora of repeaters especially for long-haul communication

Corresponding Author: Supian, L.S., Department of Electrical, Electronics and Systems Engineering, Faculty of Engineering and Built Environment Universiti Kebangsaan 43600 UKM Bangi, Selangor, Malaysia Tel: +603-89216312 
system applications (Khare, 2004). In general, communication system using optic cables are limiting the bandwidth up to $2 \mathrm{Gbps}$ (Horak, 2007). Polymer optical fibers basically made up of plastic which gives the property of insulator and is useful for many applications. It makes the signal that traversed along the fiber free from radio-frequency interference or RFI and electromagnetic interference or EMI. POFs do not pick or propagate electromagnetic pulses or known as FMPs (Khare, 2004). Moreover, in contrast to copper cables, signal transmitter through POF cannot be obtained without physically intruding the fiber. The signals that propagate along the fibers also are protected from interference and coupling with other communication channels, be it electrical or optical. Therefore, POF does offer a high degree of signal security not only for short communication system but also for long distance communications. Moreover, in contrast to the fragile nature of glass fiber, POFs are strong yet flexible. Thus, nowadays, POF is widely used in many applications especially in fiber-to-the-home network and in automobile system. However, in the simplest kind of communication system, information can be transmitted from one point to another in one direction or both directions which in either case only requires a transmitter and a receiver. Multiplexing is a technique of combining two or more signals to be transmitted over the same communication link and it can be achieved optically. At a receiver-end, a demultiplexer splits the combined signals to be sent to its destination. There is hardly any limitation of bandwidth utilization through WDM technique (Romeiser, 2006). Wavelength Division Multiplexing or WDM is a technique to upgrade the capacity of installed point-topoint fiber optic links. Wavelength multiplexer is used to combine different signal wavelengths and demultiplexer is used to separate the combine wavelengths to its original wavelength. The prime components used in the systems are basically couplers or splitter, multiplexer and demultiplexer. Hand-made $1 \times 3$ POF splitter is used in this study (Ab-Rahman et al., 2009). This study will focus on demultiplexer that utilizes the technique of wavelength filter using transparent colored thin-film. This method is not only inexpensive, easy to develop but then is efficient for data transmission in WDM-POF short-haul communication system. Samples of thin-films are selected and experimentally tested to obtain the thinfilm that gives optimum transmission of the desired signal and block any other unwanted signals. This study focus on the Red thin-film selections and the performance of each samples selected are characterized and analyzed. Spectral energy distribution provided with each thin-film is analyzed for the first stage of selecting the thin-films that could give optimum transmission. Efficiency of signal transmission and the loss percentage of the thin-film is measured based on insertion loss and power output using power meter.

Specification of design: The hand-made color thin-film based demultiplexer is an optical device which at the end of an optical fiber attached with a plug assembly; the film is adhered on the surface of the polished fiber. A coupler is connected to the fiber that carried several signals and combined them into one single fiber which functions as a multiplexer. The signals that went through the splitter will then be separated at the end of a fiber where the thin film will filter the wavelength that pass through it. For example, the red colored thin film will only let the wavelength of around $650 \mathrm{~nm}$ to pass through the film or otherwise absorbs the wavelength or filtered. The film that has been attached to the end of the plug assembly or socket using epoxyresin and fiber is then secured with another socket joint or single position bulkhead receptacle that functions as connector that connects another fiber at the other end which leads to the receiver-end. Thin layer of epoxyresin is applied to the edge of the socket and it is important to make sure that the glue does not spread too much on the fiber's surface in order to avoid any excess loss due to the fabrication handling. In complete setup system, each of red, green and blue film is attached to an end of a splitter to demultiplex the signals back to the original ones and send the signals to the respective end-receivers.

\section{MATERIALS AND METHODS}

Color thin-films are fully utilized to develop the demultiplexer in this study due to its properties of having certain layers and substrate that allow only certain wavelengths to pass through which will act perfectly as a filter. Initial stage requires the selections of thin films based on properties displayed on the 'Spectral Energy Distribution' (SED) where only red thin-films with higher transmission range of that particular wavelength are chosen.

Approach and method: To develop the filtering device, the first stage starts from selection of thin films colored filters. From various colored films used, S.E.D curves Roscolux Color Filters: Product Description, 1998 were analyzed and energy spectrum curves that state the high peak of transmission percentage of wavelength around $650 \mathrm{~nm}$ for red film low base or zero transmission for other unwanted wavelength range 
will be selected. Several samples are chosen each with different wavelength transmission peak. Each of the red thin-film samples are attached to fibers of the same length using mixture of epoxy-resin. This approach is to specify the selection of the thin-films as to which colored film gives low-loss transmission by measuring the insertion loss using power meter. Small loss of insertion losses $(\mathrm{dBm})$ and optimum power output $(\mu \mathrm{W})$ of the thin-film are preferred. Each of the film is then attached to the end of the fibers which initially the end of each fiber will be initially inserted inside a socket. Then surface of the fiber is refined by rubbing the end surface of the fiber with sand papers. Two kinds of sand papers were used. The first one is to level the rough surface until it is smooth. The second one is to make the surface finer so that the light passes through it is fully transmitted. This step is crucial so that the surface of the contact between fiber and thin-film is smooth so that the losses due to the fiber are kept as low as possible. A square cut-up red thin-film will is glued using strong adhesive of epoxy-resin mixture. Only small layer of glue is applied to the edge of the plug assembly or socket that connected to the fiber. This method has to be done implicitly so that no excessive glue will spread around the surface of the fiber that can lead to losses of transmission. A position bulkhead receptacle is used to secure the film attached to the socket. The other end of the connector will have another short optical connected to it where this part will be used to connect to the power meter. Before the measurement begins, the short fiber that connected to the connector is measured for the short optical fiber initial loss. Red LED source is used to transmit red wavelength $(\lambda=650 \mathrm{~nm})$ through the fiber and measurement are taken as to which film give the better reading. Low insertion losses $(\mathrm{dBm})$ and higher power output $(\mu \mathrm{W})$ is favored. All the colored films were tested using 3-meter length of fibers as to test the effectiveness of the transmission. Several factors could also contribute to the losses such as excessiveness of glue spread or the bendiness of the fibers and the length of the fibers used. If other factors were considered ideal, the thin film could possibly made as an effective filter for demultiplexer device for longer distance.

Characterization: Eleven red thin-film samples that show high transmission curves based on energy spectrum distribution are tested experimentally using 3meter length fibers for all red thin-films. Characterization testing is done for all the samples using power meter where initially, the power meter was calibrated by connecting the power meter with a short fiber of $20 \mathrm{~cm}$ length. Insertion loss could have rooted from fiber optic itself, the connector or splices. The initial reference value of the short optical fiber test is recorded. The power meter has to be operated within the wavelengths used in the transmission system. The power meter and data transmission sources utilized the same source that is the LED. Measurement parameters that were taken are insertion loss $(\mathrm{dBm})$, output power $(\mathrm{dBm})$ and output power $(\mu \mathrm{W})$. The differences of losses displayed and the initial reading are calculated to get the value of insertion loss. The output power $(\mu \mathrm{W})$ recorded were analyzed to evaluate the efficiency of the fiber optic and the effectiveness of the red thin films chosen. The study will only focus on the characterization analysis for same source which is the red LED source and different types of red thin-films. Red LED is injected through the fibers that were connected to the Red thin film samples. The insertion loss readings were recorded and analyzed using simulation graphs and plotted against the types of red thin-film samples. Samples that show the low insertion loss and small decrease of power output are chosen to develop a demultiplexer with performance of optimum efficiency. High output power $(\mu \mathrm{W})$ leads to optimum efficiency.

\section{RESULTS}

It can be seen that various loss depicted as shown in Fig. 1-7. Sample fifth and tenth show the best results. Low loss of insertion loss for red LED source injected through the thin-film for sample fifth and tenth while maintaining high power output compared to other red thin-films.

After the ten samples of Red thin films were injected with Red LED source, the eighth sample of red thin-film as in Fig. 1. Shows the biggest insertion loss.

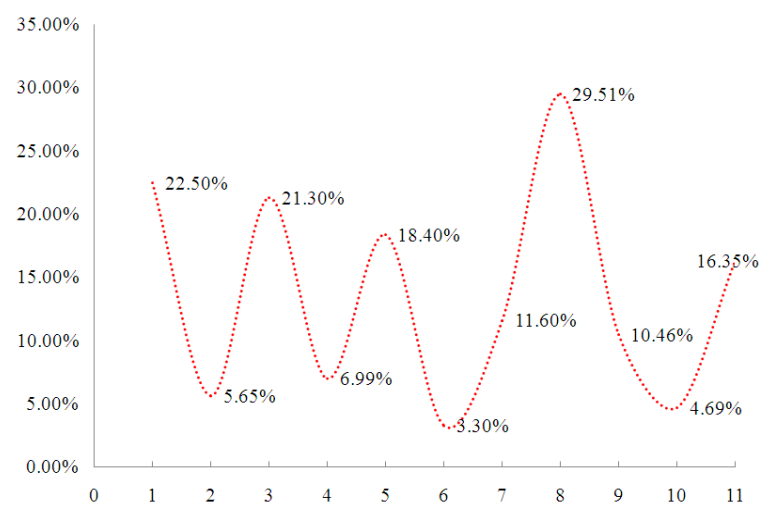

Fig. 1:Decrease percentage of output power $(\mu \mathrm{W})$ for red thin-film samples injected with red LED source 
Am. J. Applied Sci., 9 (2): 180-185, 2012

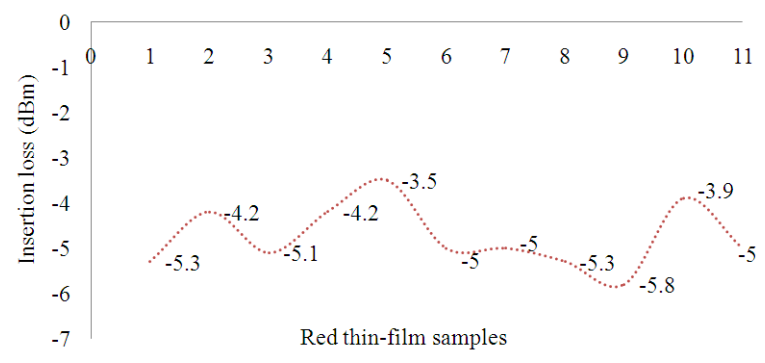

Fig. 2: Insertion loss for red thin-film samples injected with red LED source

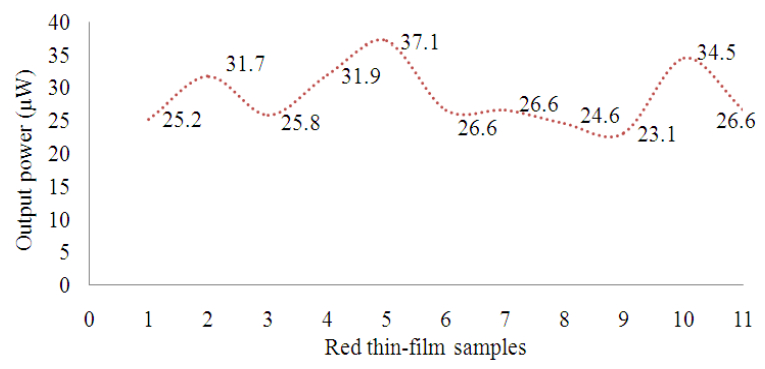

Fig. 3: Output power for red thin-film samples injected with red LED source

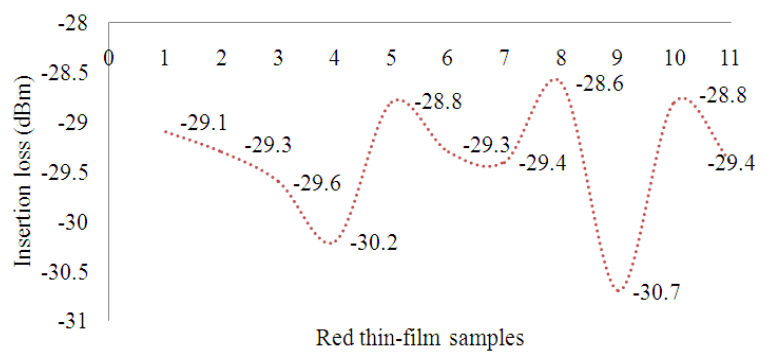

Fig. 4: Insertion loss for red thin-film samples injected with green LED source

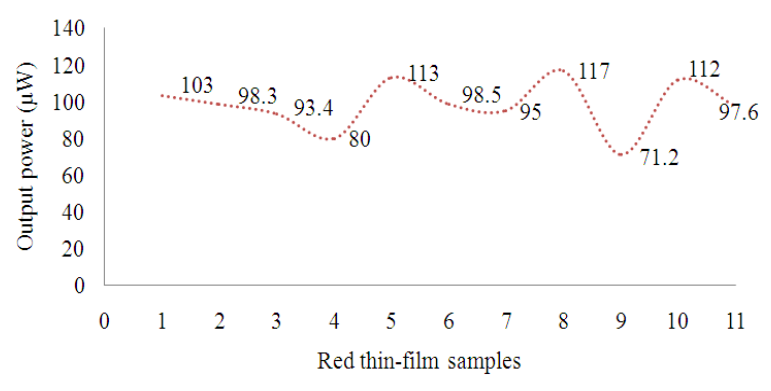

Fig. 5: Output power for red thin-film samples injected with green LED source

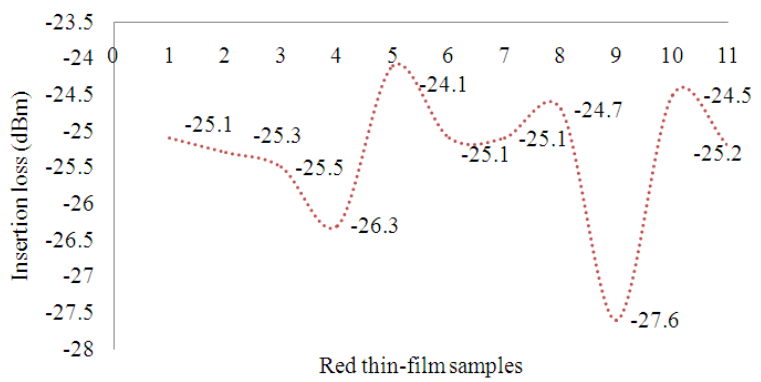

Fig. 6:Insertion loss for red thin-film samples injected with blue LED source

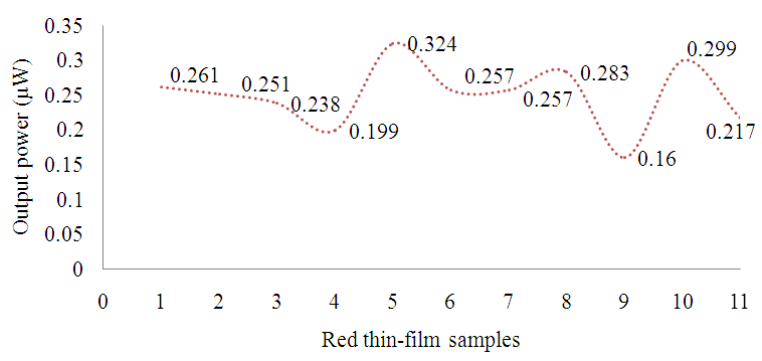

Fig. 7: Output power for red thin-film samples injected with blue LED source

Then, Blue LED and Green LED sources were injected through it. Ironically, only small insertion losses were recorded when both Blue and Green light sources pass through the film. This is due to the ineffectiveness of the eighth sample to absorb or block the Blue and Green wavelength to pass through it. It is known that the eighth sample is not effective to make it as a filter and demultiplexer due to its inability to block any other unwanted signals or wavelengths. The fifth red thin-film sample as in Fig. 1. Is analyzed for its efficiency as a filter. Same process was done and the result shows that $0.2 \mathrm{dBm}$ of insertion loss was recorded as the Red LED was injected through it. A considerable low insertion losses were recorded when Blue and Green LED source injected through the fiber and the film. The decrease of power output value can be seen by the characterization analysis. Higher losses of power output for Blue and Green LED sources were recorded compared to the Red LED source. Only the signal or wavelength of the Red LED source was allowed to be transmitted through the film. This can be referred to the low loss of power output $(\mu \mathrm{W})$.

\section{DISCUSSION}

The weak connection link between the fiber and the LED light source and bending losses could be the 
factor that contributed to the highest loss as depicted in the figures shown. The attenuation of the signal transmitted is caused by the spreading of epoxy-resin glue throughout the fiber's surface when the film was attached to the fiber and socket. The layer of the glue attenuates the signal that was transmitted through the fiber and the thin film. The fifth red thin-film sample as in Fig. 1. Shows small loss when the Red LED is injected through the fiber and thin-film. The factor that contributed to the loss could be the layer of epoxy-resin glue that spread onto the fiber's surface that attenuate the signal transmitted through the film. Higher losses of power output for Blue and Green LED sources were recorded compared to the Red LED source as shown in Fig. 8 and 9 when the Red LED source is injected through red, blue and green thin-films.

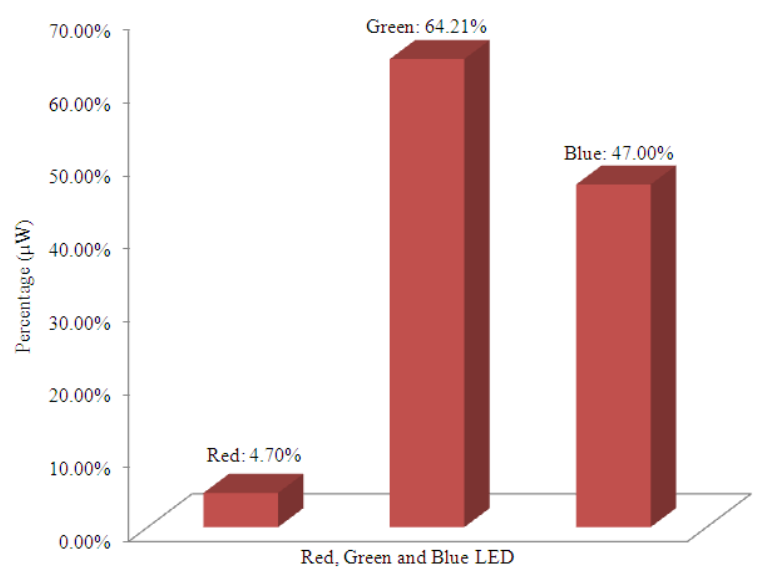

Fig. 8: Decrease percentage of power output for red, green and blue LED source injected to the eighth red thin-film sample

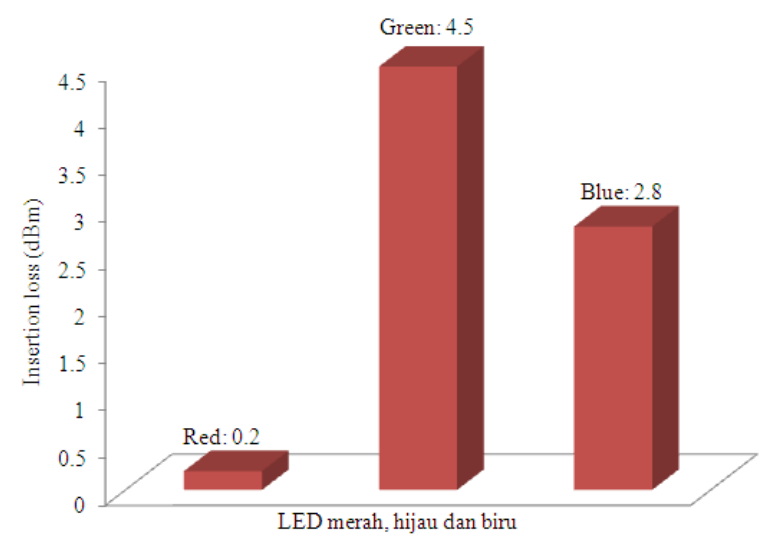

Fig. 9: The increase of insertion loss for red, green and blue LED sources injected through eighth red thin-film sample
The Blue or Green signal or wavelengths are mostly absorbed by the film and only small percentage of insignificant signal went through the film. From the characterization analysis, the eight red thin-film sample is an effective thin colored film that could be used as a filter and thus to develop demultiplexer. Losses that led to the insertion loss and high decrease of power output could be contributed by factors such as the thick layer of the epoxy-resin glue, the uneven surface of the fiber that attached to the films or the bending losses inside the fibers. Improvement for the attachment of thin film onto the surface and the connection link of the system is considered for future work as to achieve better results and therefore to develop a much effective demultiplexer using thin film colored filter.

\section{CONCLUSION}

Through this experimental test and characterization analysis, colored thin films have been proved to effectively filter out any undesired signals or wavelengths that pass through it. Only intended signal could be sent through the fiber and the film and hence channeled out to the receiver-end. It is important to note that not all the films can filter out any unwanted wavelength. Some colored films have higher transmission percentage but the film could possibly let other undesired wavelength to get through the film and ruin the effectiveness of the filter. Few thin-films block most of the undesired wavelengths; however, the thinfilms also block most of the transmission of the desired wavelength which contributes to the poor performance of the film as a wavelength filter. Characterization tests and analysis are done on red thin-film samples after being analyzed using energy spectrum distribution. The characterization is made by measuring the films properties of insertion loss $(\mathrm{dBm})$ and power output $(\mu \mathrm{W})$ when the films are connected to polymer optic fiber. Results show that certain films do function as an effective filter in WDM-POF system. Small insertion loss of the red LED source and the red thin-films and small decrease of power output values are preferred. Whereas higher insertion loss and huge decrease of power output for blue and green LED source and red thin-films are preferred. Samples used optical fibers of fixed length of 3-meter long and future improvement will be considered so that the device can give better performance for longer distance. Factors that contributed to the losses are also considered for future works. This filtering device is highly recommended as an alternative for developing demultiplexer in WDMPOF system as the cost is low and the development of the device is easy. Optimum performance is proven 
when the end-receivers that have been connected to the device included the Ethernet connectivity and the display of video image received the signals as desired.

\section{ACKNOWLEDGMENT}

The researchers would like to thank Spectech Lab group, University Kebangsaan Malaysia for the support of this project.

\section{REFERENCES}

Ab-Rahman, M.S., H. Guna and M.H. Harun, 2009. Cost-effective $1 \times 12$ POF-based optical splitters as an alternative optical transmission media for multipurpose application. Int. J. Comput. Sci. Netw. Secu., 9: 72-78.
Horak, R., 2007. Telecommunications and Data Communications Handbook. 1st Edn., John Wiley and Sons, Hoboken, N.J., ISBN: 0470041412, pp: 791.

Khare, R.P., 2004. Fiber Optics and Optoelectronics. 1st Edn., Oxford University Press, New Delhi, ISBN: 0195669304, pp: 420.

Romeiser, M., 2006. Optical fibers and Rf: A Natural Combination. 1st Edn., Prentice-Hall of India, New Delhi, India, ISBN: 8120330153, pp: 288. 